\title{
From the nonlinear Fokker-Planck equation to the Vlasov description and back: Confined interacting particles with drag
}

\author{
A. R. Plastino, ${ }^{1}$ E. M. F. Curado, ${ }^{2}$ F. D. Nobre, ${ }^{2}$ and C. Tsallis ${ }^{2,3,4}$ \\ ${ }^{1}$ CeBio y Secretaría de Investigación, Universidad Nacional del Noroeste de la Província de Buenos Aires, \\ UNNOBA-Conicet, Roque Saenz Peña 456, Junin, Argentina \\ ${ }^{2}$ Centro Brasileiro de Pesquisas Físicas and National Institute of Science and Technology for Complex Systems, \\ Rua Xavier Sigaud 150, 22290-180, Rio de Janeiro-RJ, Brazil \\ ${ }^{3}$ Santa Fe Institute, 1399 Hyde Park Road, Santa Fe, New Mexico 87501, USA \\ ${ }^{4}$ Complexity Science Hub Vienna, Josefstadter Strasse 39, 1080 Vienna, Austria
}

(Received 16 December 2017; published 14 February 2018)

\begin{abstract}
Nonlinear Fokker-Planck equations endowed with power-law diffusion terms have proven to be valuable tools for the study of diverse complex systems in physics, biology, and other fields. The nonlinearity appearing in these evolution equations can be interpreted as providing an effective description of a system of particles interacting via short-range forces while performing overdamped motion under the effect of an external confining potential. This point of view has been recently applied to the study of thermodynamical features of interacting vortices in type II superconductors. In the present work we explore an embedding of the nonlinear Fokker-Planck equation within a Vlasov equation, thus incorporating inertial effects to the concomitant particle dynamics. Exact time-dependent solutions of the $q$-Gaussian form (with compact support) are obtained for the Vlasov equation in the case of quadratic confining potentials.
\end{abstract}

DOI: 10.1103/PhysRevE.97.022120

\section{INTRODUCTION}

A variety of phenomena in complex systems [1-8] has been recently addressed using nonlinear Fokker-Planck equations (NLFPEs) [9]. Specific applications range over several subjects, including type II superconductors [10], granular media [11], and self-gravitating systems $[12,13]$. A NLFPE determines the evolution of a time-dependent density $\rho$, defined on an appropriate configuration space. One of the most studied and well-known instances of the NLFPE involves two components: a power-law, nonlinear diffusion $[14,15]$ one, and a linear drift term. In many of the aforementioned applications, the density $\rho$ should be regarded as a real physical density describing the distribution of particles in configuration space (as opposed to a statistical ensemble probability density) $[7,16]$. In these types of settings, the nonlinear diffusion term provides a convenient, effective description of the interaction between the particles of the system, while the drift term accounts for the effects of other external forces acting upon them. Nonlinear diffusion also provides useful phenomenological models in population dynamics for the spread of interacting organisms [17-19] and for the spread of energy in strongly nonlinear disordered lattices [20]. The NLFPEs recently considered in the literature are endowed with several mathematically interesting and physically relevant features. They satisfy an $H$ theorem involving a free-energy-like quantity defined in terms of the $S_{q}$ nonadditive entropic functionals [21-24]. These entropic measures have been applied to the study of diverse complex systems [25-28]. In some important instances, the NLFPEs have exact analytical solutions of the $q$-Gaussian form, which can be regarded as maximum entropy densities optimizing the $S_{q}$ entropies under suitable, simple constraints $[23,25]$. In fact, there is a profound relationship between the nonlinear Fokker-Planck dynamics and the thermostatistical formalism associated with the $S_{q}$ entropies. Although this connection was originally pointed out more than 20 years ago [29], the investigation of its multiple physical implications has only blossomed in recent years (see, for example, Refs. [1,16,30-33] and references therein). Indeed, the $S_{q}$-NLFPE relationship [29] is at the core of one of the best understood (especially from the analytical point of view $[7,10,16])$ mechanisms explaining the phenomenological success of the $q$-thermostatistical theory [25] in describing various types of complex systems. A notable example of these lines of development is given by recently reported experimental results on granular media [11], verifying, within a $2 \%$ error and for a considerable range of experimental situations, a scale relation predicted in 1996 on the basis of the theoretical analysis of the $q$-Gaussian solutions of the NLFPE [34].

The Vlasov equation [35-45] is an important tool in several branches of physics, including galactic dynamics [35], plasma physics [36,43], collective dynamics in nuclear matter and atomic nuclei [44], and the thermostatistics of classical spinlike systems [45]. It was first introduced by Jeans [37] for studying the dynamics of self-gravitating stellar systems like galaxies and, much later and independently, reintroduced by Vlasov in the context of plasma physics [38]. The Vlasov dynamics is central to one of the most important applications of $S_{q}$ thermostatistics, which is the description of the dark matter haloes of galaxies [40-42]. Vlasov dynamics also played a distinguished role at the beginning of the development of $S_{q}$ thermostatistics. The realization that some of the basic solutions of the Vlasov-Poisson system of equations in stellar dynamics, the polytropic spheres, are actually $S_{q}$-maximum entropy distributions, suggested a relation between the $S_{q}$ 
entropy and self-gravitating systems (see Ref. [46] and references therein) and, more generally, with systems exhibiting long-range interactions [25]. This connection constitutes nowadays one the most productive fields of research related to the $S_{q}$ thermostatistics $[47,48]$. In the present work we provide a link between two types of dynamics associated with the $S_{q}$ thermostatistics: the Vlasov dynamics of $N$-particle systems exhibiting $q$-Gaussian space-velocity distributions, and the dynamics of overdamped, interacting particles described by the power-law NLFPEs.

\section{THE NONLINEAR FOKKER-PLANCK EQUATION}

The starting point of the present work is the power-law NLFPE

$$
\frac{\partial P}{\partial t}=\mathcal{D} \frac{\partial^{2}}{\partial x^{2}}\left[P\left(\frac{P}{P_{0}}\right)^{1-q}\right]-\frac{\partial(P K)}{\partial x},
$$

where $P(x, t)$ is a time-dependent density, $\mathcal{D}$ is a diffusion constant $[\mathcal{D}(2-q)>0], K(x)$ is a drift force, and $q$ is a real parameter characterizing the (power-law) nonlinearity appearing in the diffusion term. The density $P$ and the constant $P_{0}$ have dimensions of inverse length. From now on, when we speak about the NLFPE we mean Eq. (1). This equation is often written in terms of a dimensionless quantity $f(x, t)=P / P_{0}$, having then the form $\frac{\partial f}{\partial t}=\mathcal{D} \frac{\partial^{2}\left(f^{2-q}\right)}{\partial x^{2}}-\frac{\partial(f K)}{\partial x}$. However, for our present purposes it will be convenient to work with the dimensional density $P(x, t)$. When dealing with one spatial dimension the drift force $K(x)$ can always be derived from a potential function $U(x), K(x)=-d U / d x$. Here we have followed the standard terminology used in the literature on the Fokker-Planck equation (both linear and nonlinear), where $K$ is called a "force" and $U$ a "potential." It is important to point out, however, that $K$ does not have dimensions of force, nor does $U$ have dimensions of energy. These two quantities are, nevertheless, respectively proportional to a force and a potential energy having the right dimensions. We shall come back to this issue in Sec. III.

The NLFPE then admits a stationary solution of the $q$ exponential form [25],

$$
\begin{aligned}
P_{q}(x) & =P_{0} A \exp _{q}[-\beta U(x)] \\
& =P_{0} A[1-(1-q) \beta U(x)]_{+}^{\frac{1}{1-q}},
\end{aligned}
$$

where $A$ and $\beta$ are positive constants to be determined, and the $q$-exponential function, $\exp _{q}(z)=[1+(1-q) z]_{+}^{\frac{1}{1-q}}$, is equal to zero whenever $1+(1-q) z \leqslant 0$. One finds that the ansatz given by (2) constitutes a stationary solution of the NLFPE (1) provided that the constants $A$ and $\beta$ comply with $(2-q) \beta \mathcal{D}=A^{q-1}$. We shall assume that the potential function $U(x)$ is such that the stationary distribution $P_{q}(x)$ has a finite norm, that is, $\int P_{q}(x) d x=I<\infty$ (we shall not assume that the norm $I$ is equal to 1 ). The specific conditions required for $P_{q}$ to have a finite norm (in particular, the range of allowed $q$-values) depend on the particular form of the potential function $U(x)$. Since in many physical applications the NLFPE describes a physical density (as opposed to a probability density) we assume that its solution $P(x, t)$ has a finite norm, but not necessarily that it is normalized to 1 .
The stationary density $P_{q}(x)$ can be regarded as a distribution optimizing the nonadditive $q$-entropic functional

$$
S_{q}[P]=\frac{k}{q-1} \int P\left[1-\left(\frac{P}{P_{0}}\right)^{q-1}\right] d x,
$$

under the constraints corresponding to the norm and the mean value of the potential $U(x)$ [25,29]. In (3) $k$ is an appropriate constant determining the units (and dimensions) of $S_{q}$. In the limit $q \rightarrow 1$ the standard linear Fokker-Planck equation,

$$
\frac{\partial P}{\partial t}=\mathcal{D} \frac{\partial^{2} P}{\partial x^{2}}-\frac{\partial(P K)}{\partial x},
$$

is recovered, and the $S_{q}$ maximum entropy stationary density (2) coincides with the well-known exponential, BoltzmannGibbs-like density,

$$
P_{B G}(x)=\frac{1}{Z} \exp \left[-\frac{1}{\mathcal{D}} U(x)\right] .
$$

In this limit the condition that has to be verified by the parameters appearing in the stationary solution reduces to $\beta \mathcal{D}=1$, becoming independent of the normalization constant $A$. The density $P_{B G}(x)$ is normalized to one if $Z=$ $\int \exp \left[-\frac{1}{\mathcal{D}} U(x)\right] d x$, and it maximizes the Boltzmann-Gibbs entropy $S_{B G}=-k \int P \ln \left(P / P_{0}\right) d x$ under the constraints of normalization and the mean value $\langle U\rangle=\int P U d x$ of the potential $U(x)$.

As already mentioned, the power-law NLFPE complies with an $H$ theorem in terms of a free-energy-like quantity based on the $S_{q}$ entropies,

$$
\frac{d}{d t}\left[(\mathcal{D} / k) S_{q^{*}}[P]-\langle U\rangle\right] \geqslant 0 .
$$

Here the functional $S_{q^{*}}$ is characterized by the entropic in$\operatorname{dex} q^{*}=2-q$. Notice that $q^{*}$ coincides with the exponent appearing in the Laplacian term of the NLFPE.

In the present work we are going to deal mostly with instances of the NLFPE corresponding to integer values of the parameter $q$. In these cases, the power-law NLFPE can be simplified by absorbing into the diffusion constant the factor $P_{0}^{q-1}$ appearing within the Laplacian term in (1). In other words, defining the new constant $D=\mathcal{D} P_{0}^{q-1}$, the NLFPE can be cast into the simpler form,

$$
\frac{\partial P}{\partial t}=D \frac{\partial^{2}\left(P^{2-q}\right)}{\partial x^{2}}-\frac{\partial(P K)}{\partial x} .
$$

It should be emphasized that, although (7) has the form of the NLFPE for a dimensionless density, we are here still dealing with a dimensional density $P$. On the other hand, the constant $D$ appearing in (7) does not have the usual dimensions of a diffusion constant. The stationary solution of (7) has the form $A[1-(1-q) \beta U(x)]_{+}^{\frac{1}{1-q}}$, with $(2-q) \beta D=A^{q-1}$. Setting $k=P_{0}^{q^{*}-1}$ for the constant $k$ appearing in the entropy $S_{q^{*}}$, the $H$ theorem satisfied by (7) is given by

$$
\frac{d}{d t}\left[D S_{q^{*}}[P]-\langle U\rangle\right] \geqslant 0,
$$

which can be regarded as a generalized free-energy $H$ theorem [22]. In order to derive (8) one has to take into account the conservation of the norm $\frac{d}{d t} \int P d x=0$. 


\section{FROM THE POWER-LAW NLFPE TO A VLASOV EQUATION}

As discussed in Ref. [7], the NLFPE can be interpreted as describing the behavior of the spatial density of a set of particles interacting via short-range interactions, and performing overdamped motion while confined by an external potential $U(x)$. In this description of that many-particle system's dynamics the inertial effects are neglected, and the evolution of the system is followed only in configuration (position) space. It is a natural next step to relax the overdamping approximation, thus including inertial effects, and find out if the close relation between these kinds of systems and the $S_{q}$-based thermostatistics still holds. That is, in a nutshell, the main purpose of the present work.

Let us consider a system of particles of mass $m$ interacting via short-range, repulsive forces, and moving under the effects of a drag force originating in a resisting medium, and of a confining potential $W$. We are going to consider a manybody system in one spatial dimension. The total force on an individual test particle has then three components: the force due to the interaction with the other particles, the drag force $-\alpha \dot{x}$ (with the constant $\alpha>0$ ), and the force due to the potential $W$. Since the particles interact via short-range repulsive forces, only close neighbors contribute to the force acting on a given particle. Intuitively one expects (we provide below a more detailed analysis of this issue) that the force on a particle due to interactions with other particles is of the form $F_{\text {int }} \propto n_{\text {left }}-n_{\text {right }}$, where $n_{\text {left,right }}$ are the number of neighbors to the left and right of the test particle that lie within the range of the interaction force. If this force satisfies some physically reasonable assumptions, the above heuristic ideas can be put in a precise form, yielding $F_{\text {int }}=-2 B \frac{\partial \tilde{\rho}}{\partial x}$, where $\tilde{\rho}(x)$ is the spatial density of particles and $B>0$ is a constant characterizing the strength of the interaction between particles [here we use the notation $\tilde{\rho}(x)$ to denote the spatial density of particles because, later in the paper, we are going to use $\rho(x, v)$ to designate the space-velocity distribution of particles; see explanation before Eq. (10)]. It is worth analyzing the force acting on one particle, due to the interaction with the other particles, in more detail (see Ref. [7] for a full discussion). Let $\mathcal{B}\left(\left|x^{\prime}-x\right|\right)\left(x^{\prime}-x\right) /\left|x^{\prime}-x\right|$ be the force on a particle located at $x$ due to a particle located at $x^{\prime}$, where $\mathcal{B}\left(\left|x^{\prime}-x\right|\right) \geqslant 0$ represents the strength of the short-range (repulsive) force between the two particles. We assume that $\mathcal{B}$ is a smooth function of the distance $r=\left|x^{\prime}-x\right|$ between the particles, which decays fast enough with $r$, so that the integral $\int_{0}^{\infty} r \mathcal{B}(r) d r$ converges. Moreover, the short-range nature of the force means that the range of distances over which $\mathcal{B}(r)$ is appreciably different from zero is small compared with the natural length scales of the system. In particular, over the range of distances where $\mathcal{B}$ differs substantially from zero the spatial density $\tilde{\rho}$ can be approximated as $\tilde{\rho}\left(x^{\prime}\right)=\tilde{\rho}(x)+\left(x^{\prime}-x\right)(\partial \tilde{\rho} / \partial x)($ see Ref. [7] for a detailed discussion). Using this approximation, the force acting on a particle located at $x$, due to the interaction with the other particles, can be written as

$$
F_{\text {int }}=-2 B \frac{\partial \tilde{\rho}}{\partial x},
$$

with $B=\int_{0}^{\infty} r \mathcal{B}(r) d r$. This form of the force $F_{\text {int }}$ acting on a test particle is formally equivalent to the one resulting from an interaction potential between particles located at $x_{1}$ and $x_{2}$ of the form $V\left(x_{1}, x_{2}\right)=2 B \delta\left(x_{2}-x_{1}\right)$, where $\delta(x)$ is Dirac's delta function. The equation of motion of one particle, including the effects of the external confining potential $W$ and a drag force $-\alpha \dot{x}$, is

$$
m \ddot{x}=-2 B \frac{\partial \tilde{\rho}}{\partial x}-\frac{\partial W}{\partial x}-\alpha \dot{x} .
$$

In the regime of overdamped motion (that is, when the inertial term $m \ddot{x}$ is much smaller than the other terms in the particle's equation of motion) one has

$$
\dot{x}=-\frac{2 B}{\alpha}\left(\frac{\partial \tilde{\rho}}{\partial x}\right)-\frac{1}{\alpha}\left(\frac{\partial W}{\partial x}\right) .
$$

The particle density $\tilde{\rho}(x, t)$ describing a system of interacting particles that move according to (11) obeys the continuity equation,

$$
\frac{\partial \tilde{\rho}}{\partial t}=\frac{2 B}{\alpha} \frac{\partial}{\partial x}\left(\tilde{\rho} \frac{\partial \tilde{\rho}}{\partial x}\right)+\frac{1}{\alpha} \frac{\partial}{\partial x}\left(\tilde{\rho} \frac{\partial W}{\partial x}\right),
$$

which, after the identifications $D \rightarrow \frac{B}{\alpha}$ and $U \rightarrow \frac{W}{\alpha}$, reduces to the $q=0$ instance of the NLFPE in (7):

$$
\frac{\partial \tilde{\rho}}{\partial t}=D \frac{\partial^{2}}{\partial x^{2}}\left(\tilde{\rho}^{2}\right)+\frac{\partial}{\partial x}\left(\tilde{\rho} \frac{\partial U}{\partial x}\right) .
$$

The above NLFPE, with a quadratic nonlinearity within the diffusion term, is the one we are going to consider in the present work. The nonlinear diffusion term in this NLFPE is associated with two-body interactions and has been recently applied to the study of the thermostatistics of interacting vortices in type II superconductors $[7,10,16]$.

In this work we are going to assume that the drag force acting on each of the particles of the system depends linearly on the velocity of the particle. This drag force originates on the interaction between the particle and the medium in which the particles constituting the system under consideration are moving. We are not going to explicitly model this particle-medium interaction. We shall take it into account only through the aforementioned, phenomenological drag force. The procedure developed here to connect the NLFPE with the Vlasov equation needs the assumption of a linear drag. The exploration of possible extensions of this approach, valid for more general drag forces, is outside the scope of the present work. In this regard, it is worth mentioning that other types of velocity dependence for drag forces also occur in nature. In fact, interesting advances, both at the theoretical and at the experimental levels, have been recently made in the study of the drag forces experienced by test particles when moving in different types of environments [49-51]. These developments allowed for the identification of relevant scenarios, characterized by particular types of interactions between the test particle and the environment particles, leading to clear departures from linear drag.

\section{A. Embedding the NLFPE within Vlasov dynamics}

We are now going to relax the overdamped motion assumption and incorporate inertial effects to the statistical description of our system of interacting particles. In order to do that, we now describe the (evolving) state of the system by a 
time-dependent space-velocity density, $\rho(x, v, t)$. The density $\rho(x, v, t)$ has dimensions of time over squared length, so that $\rho d x d v$ is dimensionless. The density $\rho$ is normalized to the total number of particles, $N=\int \rho d x d v$, and the spatial density $\tilde{\rho}$ is obtained by integrating $\rho$ over all velocities, $\tilde{\rho}(x, t)=\int \rho(x, v, t) d v$. The equation of motion of a test particle is then given by (10), and the associated continuity equation satisfied by $\rho(x, v, t)$ constitutes the Vlasov equation corresponding to our multiparticle system,

$$
\frac{\partial \rho}{\partial t}+\frac{\partial}{\partial v}\left(\frac{F}{m} \rho\right)+\frac{\partial}{\partial x}(v \rho)=0,
$$

where $F$ is the total force acting on an individual particle,

$$
F=-2 B \frac{\partial \tilde{\rho}}{\partial x}-\frac{\partial W}{\partial x}-\alpha \dot{x} .
$$

Notice that the contribution to $F$ due to the interaction with the other particles of the system is given by the gradient of the spatial density $\tilde{\rho}$, because the interaction forces are assumed to be only position dependent (not velocity dependent). The evolution equation (14) seems to be linear in $\rho$, but it is actually nonlinear, because the force $F$ depends on $\rho$ itself. The main physical assumption behind the evolution equation (14), besides the basic one of having a large enough number of particles in each element $d x d v$ of the $(x, v)$ plane so that the system can be described by a continuous density $\rho(x, v)$, is that (15) provides a good description of the total force acting on an individual particle of the system. And, in particular, the force $F_{\text {int }}$ given in (9) accounts completely for the interaction between one particle and the rest of the particles of the system. These physical assumptions are also central to the validity of the quadratic NLFPE as an effective description of a set of repulsively interacting particles doing overdamped motion, as discussed in Ref. [7]. Indeed, the numerical evidence reported there attests to the fact that, for the type of many-particle systems discussed here, the above mentioned assumptions are actually satisfied.

\section{B. The Vlasov dynamics}

The dynamics of the system of particles under consideration is then described by the Vlasov equation,

$$
\begin{aligned}
\frac{\partial \rho}{\partial t}= & \frac{\partial}{\partial v}\left(\frac{2 B}{m} \rho \frac{\partial \tilde{\rho}}{\partial x}\right)+\frac{\partial}{\partial v}\left(\frac{1}{m} \rho \frac{\partial W}{\partial x}\right) \\
& +\frac{\partial}{\partial v}\left(\frac{\alpha}{m} v \rho\right)-\frac{\partial}{\partial x}(v \rho) .
\end{aligned}
$$

The Vlasov equation can equivalently be cast as

$$
\left(\frac{d \rho}{d t}\right)_{\text {orbit }}=\frac{\alpha}{m} \rho
$$

where

$$
\begin{aligned}
\left(\frac{d \rho}{d t}\right)_{\text {orbit }}= & \frac{\partial \rho}{\partial t}-\frac{2 B}{m}\left(\frac{\partial \tilde{\rho}}{\partial x}\right) \frac{\partial \rho}{\partial v}-\frac{1}{m}\left(\frac{\partial W}{\partial x}\right) \frac{\partial \rho}{\partial v} \\
& -\left(\frac{\alpha v}{m}\right) \frac{\partial \rho}{\partial v}+v \frac{\partial \rho}{\partial x}
\end{aligned}
$$

is the total time derivative of the density evaluated along one particle's orbit. It follows from (17) that the local space-velocity density around a given particle behaves as $\rho_{t}^{\text {(orbit) }}=\rho_{t=0} \exp (\alpha t / m)$. This implies that for $\alpha \neq 0$ the Vlasov equation (16) does not admit stationary solutions [except the trivial one where $\rho$ is equal to zero everywhere in the $(x, v)$ plane]. However, as we shall shortly see, for appropriate interaction and external potentials, $V\left(x_{1}, x_{2}\right)$ and $W(x)$, respectively, there is a stationary spatial density $\tilde{\rho}(x)$.

It is useful to consider the evolution of the total energy of the system,

$$
\begin{aligned}
E= & \frac{m}{2} \int v^{2} \rho d x d v \\
& +\int V\left(x_{1}, x_{2}\right) \rho\left(x_{1}, v_{1}, t\right) \rho\left(x_{2}, v_{2}, t\right) d x_{1} d x_{2} d v_{1} d v_{2} \\
& +\int \rho W d x d v
\end{aligned}
$$

where the first term on the right-hand-side of the above equation corresponds to the system's kinetic energy $E_{K}=$ $\frac{m}{2} \int v^{2} \rho d x d v$ and the other two terms to the potential energy, which can be expressed in terms of the spatial density $\tilde{\rho}(x, t)$ as

$$
\begin{aligned}
E_{\mathrm{pot}}= & \int V\left(x_{1}, x_{2}\right) \tilde{\rho}\left(x_{1}, t\right) \tilde{\rho}\left(x_{2}, t\right) d x_{1} d x_{2} \\
& +\int \tilde{\rho}(x, t) W(x) d x .
\end{aligned}
$$

It is possible to show that the time derivative of the total energy is given by

$$
\frac{d E}{d t}=-\alpha m \int v^{2} \rho d x d v \leqslant 0 .
$$

As expected, due to the drag forces, the total energy is a decreasing function of time. For the particular value $\alpha=0$, the system is conservative and the energy becomes an integral of motion.

For $\alpha>0$ a stationary particle configuration would correspond to a global minimum of the energy functional $E$. This minimum can be realized for a state of the system having $E_{K}=$ 0 , if the potentials $V\left(x_{1}, x_{2}\right)$ and $W(x)$ are such that there is a spatial density $\tilde{\rho}_{\min }(x)$ that minimizes the potential energy (20). This is indeed the case for the short-range repulsive interactions considered in the present case, if one considers an appropriate confining potential $W$ as, for example, a quadratic confining potential. The stationary distribution then corresponds to the space-velocity density $\rho(x, v)=\tilde{\rho}_{\min }(x) \delta(v)$. For the shortrange repulsive interactions considered here the stationary density $\tilde{\rho}_{\min }(x)$ is then given by the variational principle $\delta \int\left[B \tilde{\rho}^{2}(x)+\tilde{\rho}(x) W(x)\right] d x=0$, which, equivalently, can be recast under the guise

$$
\delta\left[B S_{2}[\tilde{\rho}]-\langle W\rangle\right]=0 .
$$

\section{The overdamped regime: Back to the NLFPE}

It is instructive to discuss briefly how the NLFPE can be recovered from the Vlasov one in the limit of overdamped motion. Integrating the Vlasov equation over all velocities, one gets

$$
\frac{\partial \tilde{\rho}}{\partial t}=-\frac{\partial}{\partial x}\left(\int v \rho d v\right)=-\frac{\partial}{\partial x}(\tilde{\rho}\langle v\rangle),
$$


where $\langle v\rangle=\frac{1}{\tilde{\rho}} \int v \rho(x, v, t) d v$ is the local average particle velocity at position $x$. In the overdamped regime particles move according to $\dot{x}=-(2 B / \alpha)(\partial \tilde{\rho} / \partial x)-(1 / \alpha)(\partial W / \partial x)$. The space-velocity density is consequently of the form

$$
\rho(x, v, t)=\delta\left(v+\frac{2 B}{\alpha} \frac{\partial \tilde{\rho}}{\partial x}+\frac{1}{\alpha} \frac{\partial W}{\partial x}\right) \tilde{\rho}(x, t),
$$

where $\delta(\cdots)$ stands for Dirac's delta function. Inserting (24) into (23) yields

$$
\frac{\partial \tilde{\rho}}{\partial t}=D \frac{\partial^{2} \tilde{\rho}^{2}}{\partial x^{2}}+\frac{\partial}{\partial x}\left(\frac{\partial U}{\partial x} \tilde{\rho}\right)
$$

which has the same form as Eq. (13), with $D=B / \alpha$ and $U=$ $W / \alpha$.

\section{Revisiting the NLFPE $H$ theorem}

The force due to interactions, $-2 B(\partial \tilde{\rho} / \partial x)$, is a conservative force that can be regarded as arising from the potential function $\phi(x)=2 B \tilde{\rho}$. Recalling the interparticle potential $V\left(x_{1}, x_{2}\right)=-2 B \delta\left(x_{1}, x_{2}\right)$, it is plain that the potential function $\phi(x)$ is just the total potential function that one particle feels in the field generated by the rest of the particles in the system, $\phi(x)=\int V\left(x, x^{\prime}\right) d x^{\prime}$. Now, in order to determine the potential energy $\Omega$ (due to the interactions) corresponding to a spatial configuration $\tilde{\rho}(x)$, we can follow the standard procedure of building $\tilde{\rho}$ incrementally, bringing successive layers $d \tilde{\rho}^{*}$ of matter "from infinity,"

$$
d \Omega=\int\left[2 B \tilde{\rho}^{*}(x) d \tilde{\rho}^{*}(x)\right] d x,
$$

yielding

$$
\Omega=2 B \int\left[\int_{0}^{\tilde{\rho}} \tilde{\rho}^{*} d \tilde{\rho}^{*}\right] d x=B \int \tilde{\rho}^{2}(x) d x .
$$

In our system, besides $\Omega$, we have another contribution to the total potential energy, due to the external potential,

$$
\langle W\rangle=\int \tilde{\rho} W d x
$$

The total potential energy, with both contributions from the interactions between particles and to the external confining potential, is then

$$
E_{\mathrm{pot}}=\Omega+\langle W\rangle=B \int \tilde{\rho}^{2}(x) d x+\int \tilde{\rho} W d x .
$$

In the overdamped regime the $N$-particle system always moves downhill in the potential energy landscape:

$$
\frac{d E_{\mathrm{pot}}}{d t} \leqslant 0 .
$$

This inequality can be recast in the equivalent form,

$$
\frac{d}{d t}\left[D S_{2}[\tilde{\rho}]-\langle U\rangle\right] \geqslant 0,
$$

which constitutes a particular case of the (generalized freeenergy) $H$ theorem found in Ref. [22], and can be proven directly from the NLFPE given by (25).

\section{EXACT TIME-DEPENDENT ANALYTICAL SOLUTIONS: THE $q$ GAUSSIAN ANSATZ}

We consider a harmonic confining potential

$$
W=\frac{1}{2} C x^{2},
$$

with $C>0$, and the $q$-Gaussian ansatz,

$$
\begin{aligned}
\rho(x, v, t)= & \rho_{0}\left[1-\left(1-q^{\prime}\right)\left(\beta_{x x}\left(x-x_{0}\right)^{2}\right.\right. \\
& \left.\left.+2 \beta_{x v}\left(x-x_{0}\right)\left(v-v_{0}\right)+\beta_{v v}\left(v-v_{0}\right)^{2}\right)\right]_{+}^{\frac{1}{1-q^{\prime}}},
\end{aligned}
$$

where $\rho_{0}, \beta_{x x}, \beta_{x v}, \beta_{v v}, x_{0}$, and $v_{0}$ are time-dependent parameters and $q^{\prime}=-1$. To have a physically sensible (that is, normalizable) distribution the parameters appearing in (33) must comply with the constraints $\beta_{x x}, \beta_{v v}>0$, and $\Delta=$ $\beta_{x x} \beta_{v v}-\beta_{x v}^{2}>0$. Under these conditions the curves of constant density in the $(x v)$ plane are ellipses, and the distribution (33) has a finite norm. The spatial density $\tilde{\rho}(x, t)$, obtained after integrating the space-velocity distribution (33) over all velocities, is

$\tilde{\rho}(x, t)$

$$
=\rho_{0} \mathcal{N}\left(\beta_{v v}\right)\left[1-\left(1-q^{\prime}\right)\left(\beta_{x x}-\frac{\beta_{x v}^{2}}{\beta_{v v}}\right)\left(x-x_{0}\right)^{2}\right]_{+}^{\frac{1}{1-q^{\prime}}+\frac{1}{2}},
$$

where

$$
\mathcal{N}\left(\beta_{v v}\right)=\int_{z_{-}}^{z_{+}} d z\left[1-\left(1-q^{\prime}\right) \beta_{v v} z^{2}\right]^{\frac{1}{1-q^{\prime}}}
$$

with $z_{ \pm}= \pm \sqrt{\frac{1}{\left(1-q^{\prime}\right) \beta_{v v}}}$. The spatial density is again a $q$-Gaussian, characterized by the parameter $q^{\prime \prime}$ given by

$$
\frac{1}{1-q^{\prime \prime}}=\frac{1}{1-q^{\prime}}+\frac{1}{2} \text {. }
$$

The appearance of different interrelated $q$-values when studying one particular system is a feature that has been observed in various applications of the $q$-thermostatistics. These $q$-values are related with each other by a set of transformations [of which (36) is a particular instance] that has been discussed in detail in Refs. [52-54]. Another scenario with multiple $q$-values is discussed in Ref. [55].

For $q^{\prime}=-1$ one has that $q^{\prime \prime}=0$, and (34) reduces to

$$
\tilde{\rho}(x, t)=\frac{\pi}{2 \sqrt{2}} \frac{\rho_{0}}{\sqrt{\beta_{v v}}}\left[1-2\left(\beta_{x x}-\frac{\beta_{x v}^{2}}{\beta_{v v}}\right)\left(x-x_{0}\right)^{2}\right]_{+} .
$$

The above spatial density has the shape of an inverted parabola, and vanishes for $\left|x-x_{0}\right| \geqslant 1 /\left(2 \beta_{v v} \Delta\right)$. The density (37) shares the parabolic form exhibited by the time-dependent solution of the NLFPE discussed in Ref. [10], although the detailed dynamics governing the former is different from the one associated with the latter. Indeed, the evolution of $\tilde{\rho}(x, t)$ arises from a Vlasov dynamics incorporating inertial effects, which are not included in the NLFPE description. Note that the spatial density is a $q$-Gaussian with a $q$-value $(q=0)$ different 
from the one corresponding to the space-velocity distribution. The norm $N$ (total number of particles in the system) is

$$
\begin{aligned}
N & =\iint \rho(x, v, t) d x d v=\int \tilde{\rho}(x, t) d x \\
& =\frac{\pi \rho_{0}}{3 \sqrt{\beta_{x x} \beta_{v v}-\beta_{x v}^{2}}} .
\end{aligned}
$$

Inserting the spatial density (37) within the Vlasov equation (16) one gets

$$
\begin{aligned}
\frac{\partial \rho}{\partial t}= & -\frac{\partial}{\partial v}\left(\frac{2 B R}{m}\left(x-x_{0}\right) \rho\right)+\frac{\partial}{\partial v}\left(\frac{C}{m} x \rho\right) \\
& +\frac{\partial}{\partial v}\left(\frac{\alpha}{m} v \rho\right)-\frac{\partial}{\partial x}(v \rho),
\end{aligned}
$$

with $R=\sqrt{2} \pi \frac{\rho_{0}}{\sqrt{\beta_{v v}}}\left(\beta_{x x}-\frac{\beta_{x v}^{2}}{\beta_{v v}}\right)$.

It can be verified after some computations that the ansatz (33) constitutes a time-dependent solution of the Vlasov equation (16), provided that the set of parameters appearing in (33) obey the set of coupled ordinary differential equations,

$$
\begin{aligned}
\dot{\rho}_{0} & =a \rho_{0}, \\
\dot{x}_{0} & =v_{0}, \\
\dot{v}_{0} & =-\frac{C}{m} x_{0}-a v_{0},
\end{aligned}
$$

and

$$
\begin{aligned}
& \dot{\beta}_{x x}=2 b \beta_{x v}, \\
& \dot{\beta}_{x v}=-\beta_{x x}+a \beta_{x v}+b \beta_{v v}, \\
& \dot{\beta}_{v v}=-2 \beta_{x v}+2 a \beta_{v v},
\end{aligned}
$$

where

$$
\begin{aligned}
& a=\alpha / m, \\
& b=\frac{C}{m}-2 \sqrt{2} \pi \frac{B \rho_{0}}{m \sqrt{\beta_{v v}}}\left(\beta_{x x}-\frac{\beta_{x v}^{2}}{\beta_{v v}}\right) .
\end{aligned}
$$

The first equation in (40) can be integrated immediately yielding $\rho_{0}(t)=\rho_{0 i} \exp (\alpha t / m)$. It transpires from the second and third equations in (40) that the center $\left(x_{0}, v_{0}\right)$ of the $q$-Gaussian solution evolves according to the equations of motion of a one-dimensional damped harmonic oscillator. For large $t$ one has $x_{0} \rightarrow 0$ and $v_{0} \rightarrow 0$.

It follows from (41) that $d \Delta / d t=2 a \Delta$, and $\Delta=$ $\Delta_{0} \exp (2 a t)$. This implies that, if the conditions that the parameters $\beta_{x x}, \beta_{v v}$, and $\beta_{x v}$ have to satisfy for normalizability are satisfied at the initial time, these conditions are also satisfied at all later times: they are preserved by the equations of motion (40)-(41). The already mentioned time dependences of $\rho_{0}$ and $\Delta$, combined with the expression (38) for the norm $N$, imply that $N$ is a constant of motion, as expected. The spatial density $\tilde{\rho}(x, t)$ associated with the time-dependent $q$-Gausian solution evolves asymptotically towards a stationary one, given by the variational principle (22), which corresponds to a minimum of the potential energy functional $E_{\text {pot }}$ given in (29). It can be verified after some algebra that the relationship that the parameters appearing in (37) have to satisfy for stationarity (that is, for the minimum of $E_{\mathrm{pot}}$ ) correspond to $b=0$ [that is, the right-hand-side of the second equation in (42) vanishes]. From

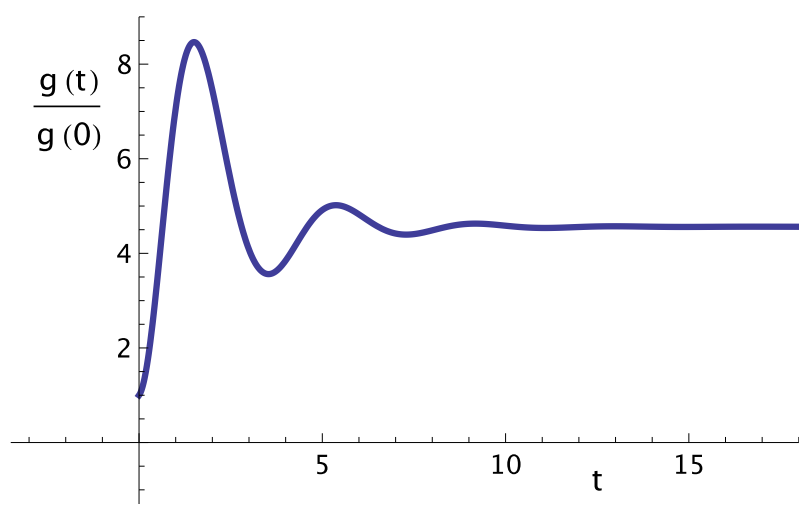

FIG. 1. The quotient $g(t) / g(0)$, where $g(t)=\beta_{v v} \exp (-2 a t)$ [see Eq. (46)] and $g(0)$ stands for the initial value of $g(t)$, is plotted against time, for $a=1, B=1, m=1, C=1, \rho_{0, i}=1$, and $\Delta_{0}=(\pi / 3)^{2}$. The quantity $g(t) / g(0)$ is dimensionless, and time is measured in units of $a^{-1}$.

the asymptotic condition $b=0$ it follows that the asymptotic behavior of $\beta_{v v}$ is given by $\beta_{v v} \approx \beta_{v v}^{(l)} \exp [2 a t]$, where $\beta_{v v}^{(l)}$ is an appropriate constant characterizing the behavior of $\beta_{v v}$ at large times. These asymptotic relations satisfied by $b$ and $\beta_{v v}$ imply, in turn, that

$$
\beta_{v v}^{(l)}=\left[\frac{2 \sqrt{2} \pi B \rho_{0, i} \Delta_{0}}{C}\right]^{\frac{2}{3}} .
$$

The asymptotic, stationary spatial density is then given by

$$
\tilde{\rho}(x)=\frac{\pi}{2 \sqrt{2}} \frac{\rho_{0, i}}{\sqrt{\beta_{v v}^{(l)}}}\left[1-2\left(\frac{\Delta_{0}}{\beta_{v v}^{(l)}}\right) x^{2}\right]_{+} .
$$

Some features of the dynamics of the system under consideration are illustrated in Figs. 1-3. The results depicted in these figures correspond to values of the system's parameters given by $a=1, B=1, m=1, C=1, \rho_{0, i}=1$, and $\Delta_{0}=(\pi / 3)^{2}$. The time evolution of the quantity $g(t) / g(0)$ [see Eq. (46)] is shown in Fig. 1. We can see that this quantity exhibits an initial oscillatory behavior, with the amplitude of the oscillations decreasing with time. For large times, $g(t) / g(0)$ approaches a constant asymptotic value. The evolution of the quantities $\beta_{x x}$ and $\beta_{v v}$ is given in Fig. 2. As shown in the inset on the left of Fig. 2, the quantity $\beta_{x x}$ stays always positive. The same happens with $\beta_{v v}$ (see the right part of Fig. 2) consistently with the fact that the quantity $\Delta=\beta_{x x} \beta_{v v}-\beta_{x v}^{2}$ is also always positive, as required for the normalizability of the space-velocity density (33). The scaled spatial density $\sqrt{g(t)} \tilde{\rho}(x)$ is depicted in Fig. 3, as a function of the scaled and centered spatial variable $\left[x-x_{0}(t)\right] / \sqrt{g(t)}$. The shape of the scaled spatial density as a function of $\left[x-x_{0}(t)\right] / \sqrt{g(t)}$ is constant in time.

To get a better understanding of the nonstationary regime associated with the evolution equations (41), it is convenient to recast those equations as a second-order ordinary differential equation in $\beta_{v v}$. We first take the time derivative of the third equation in (41). In the right-hand side of the resulting equation we substitute $\dot{\beta}_{x v}$ by the expression in the right-hand side of the second equation in (41). In the equation resulting from this 

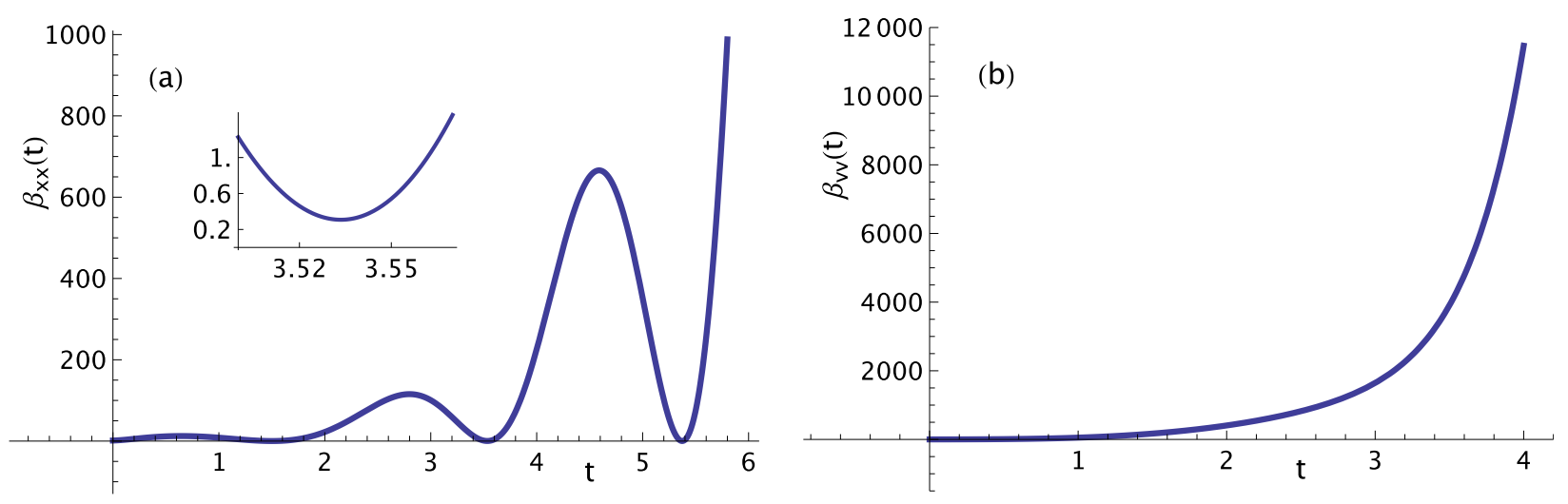

FIG. 2. Plots of $\beta_{x x}(t)$ against time (a) and $\beta_{v v}(t)$ against time (b). The values of the physical parameters characterizing the system are the same as those detailed in the caption of Fig. 1. The quantity $\beta_{x x}$ is measured in units of $\left[\frac{m a^{2}}{B}\right]^{2 / 3}, \beta_{v v}$ in units of $\left[\frac{m}{B a}\right]^{2 / 3}$, and time in units of $a^{-1}$.

substitution we replace $\beta_{x x}$ by $\left(\Delta+\beta_{x v}^{2}\right) / \beta_{v v}$. Finally, we use the third equation in (41) again, now in order to substitute $\beta_{x v}$ by an expression involving only $\beta_{v v}$ and $\dot{\beta}_{v v}$. These steps lead to the equation

$$
\ddot{\beta}_{v v}=\frac{2 \Delta}{\beta_{v v}}+a \dot{\beta}_{v v}+\frac{\dot{\beta}_{v v}^{2}}{2 \beta_{v v}}-\frac{2 C}{m} \beta_{v v}+4 \sqrt{2} \pi \frac{B \rho_{0} \Delta}{m \beta_{v v}^{1 / 2}} .
$$

The previously determined asymptotic behavior for large times, $\beta_{v v} \approx \beta_{v v}^{(l)} \exp (2 a t)$, suggests to reformulate (45) in terms of the ansatz:

$$
\beta_{v v}=g(t) \exp (2 a t) .
$$

After some calculations one then obtains the following evolution equation for $g(t)$ :

$$
\begin{aligned}
\ddot{g}= & \frac{2 \Delta_{0}}{g} \exp (-2 a t)-a \dot{g}+\frac{\dot{g}^{2}}{2 g}-\frac{2 C}{m} g \\
& +4 \sqrt{2} \pi \frac{B \rho_{0, i} \Delta_{0}}{m g^{1 / 2}} .
\end{aligned}
$$

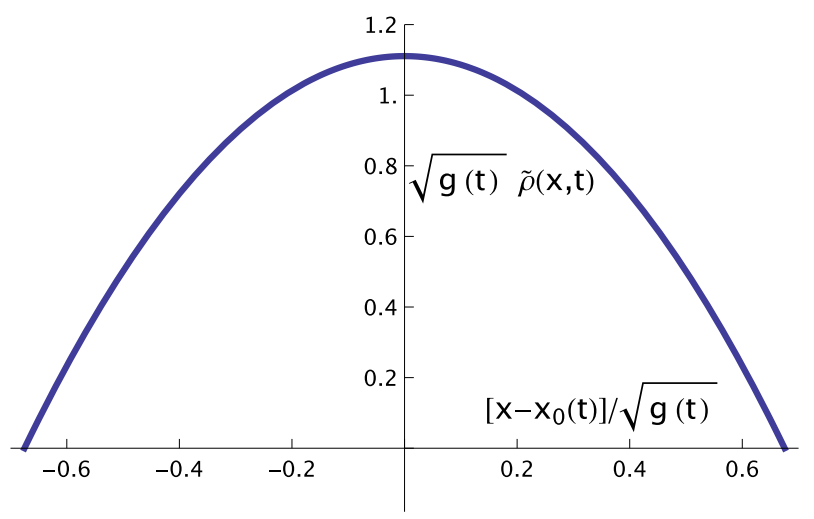

FIG. 3. Plot of the scaled spatial density $\sqrt{g(t)} \tilde{\rho}(x)$ as a function of the scaled and centered spatial variable $\left[x-x_{0}(t)\right] / \sqrt{g(t)}$. The scaled density $\sqrt{g} \tilde{\rho}$ is measured in units of $\left[\frac{m^{2} a}{B^{2}}\right]^{1 / 3}$ and the spatial coordinate $\left[x-x_{0}(t)\right] / \sqrt{g}$ in units of $\left[\frac{B^{2}}{m^{2} a}\right]^{1 / 3}$.
In order to interpret this equation of motion it is convenient to make the change of variables $z=\sqrt{g}$, yielding

$$
\ddot{z}=\frac{\Delta_{0}}{z^{3}} \exp (-2 a t)-\frac{C}{m} z+2 \sqrt{2} \pi \frac{B \rho_{0, i} \Delta_{0}}{m z^{2}}-a \dot{z} .
$$

We see that the equation of motion of $z$ can be written as $\ddot{z}=-\frac{\partial \Phi}{\partial z}-a \dot{z}$, with

$$
\Phi(z, t)=\frac{\Delta_{0}}{2 z^{2}} \exp (-2 a t)+\frac{C}{2 m} z^{2}+2 \sqrt{2} \pi \frac{B \rho_{0, i} \Delta_{0}}{m z} .
$$

The equation of motion for $z$ is formally equivalent to the equation of motion of a particle of unit mass moving in one spatial dimension under the time-dependent potential $\Phi(z, t)$ and the drag force $-a \dot{z}$. The energy of this particle, $\epsilon=$ $\frac{1}{2} \dot{z}^{2}+\Phi(z, t)$, decreases monotonically in time, since $d \epsilon / d t=$ $-\frac{a \Delta_{0}}{z^{2}} \exp (-2 a t)-a \dot{z}^{2}<0$. Consequently, at large times the value of $z$ tends towards the minimum of $\Phi$, which is at $z_{m}=\left(2 \sqrt{2} \pi B \rho_{0, i} \Delta_{0} / C\right)^{1 / 3}$, corresponding to the stationary value $\left[\beta_{v v}^{(l)}\right]^{1 / 2}$.

\section{CONSERVATIVE DYNAMICS}

Of special interest is the system obtained when $\alpha=0$, corresponding to vanishing drag, which describes a conservative $N$-particle system. In this case we have that $\Delta=\Delta_{0}=$ const and $\rho_{0}=\rho_{0, i}=$ const. The equations of motion associated with the $q$-Gaussian solution are

$$
\begin{aligned}
& \dot{x}_{0}=v_{0}, \\
& \dot{v}_{0}=-\frac{C}{m} x_{0},
\end{aligned}
$$

and

$$
\begin{aligned}
& \dot{\beta}_{x x}=2 b \beta_{x v}, \\
& \dot{\beta}_{x v}=-\beta_{x x}+b \beta_{v v}, \\
& \dot{\beta}_{v v}=-2 \beta_{x v} .
\end{aligned}
$$

The first set of equations corresponds to a one-dimensional (conservative) harmonic oscillator. The equations of motion 

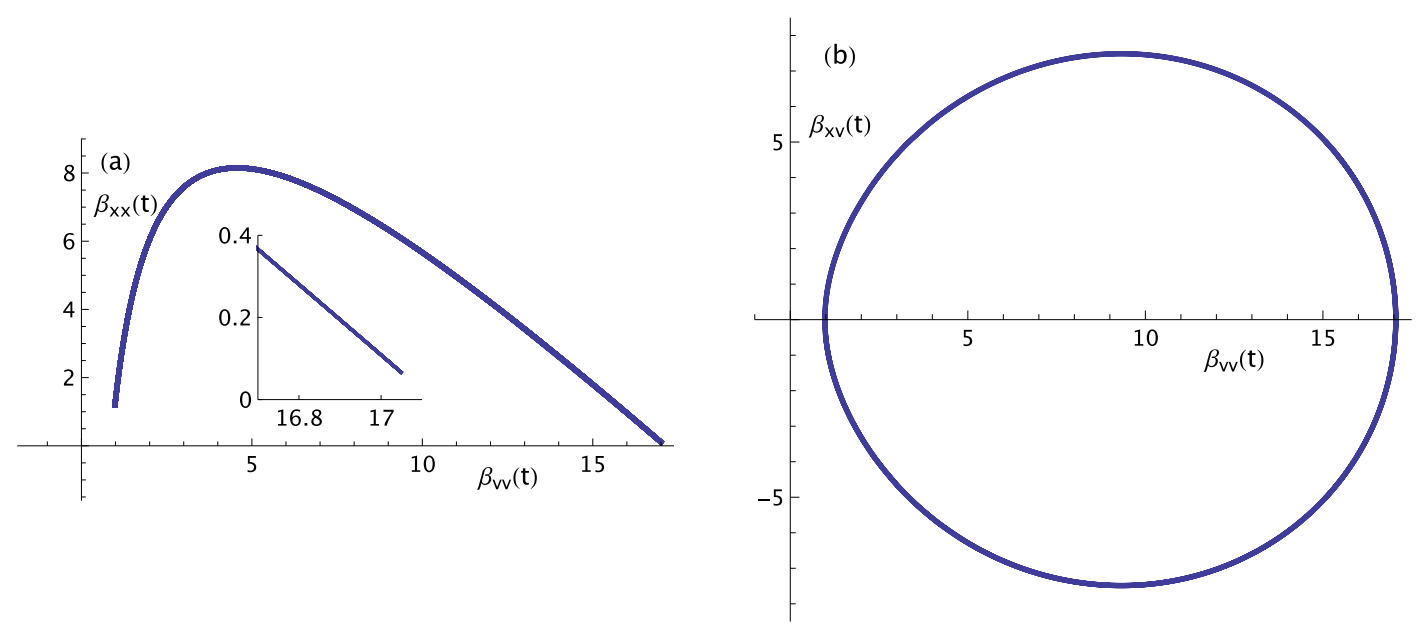

FIG. 4. Plot of $\beta_{x x}$ against $\beta_{v v}$ (a) and $\beta_{x v}$ against $\beta_{v v}$ (b) along one orbit of the conservative system $(a=0)$, with the other physical parameters characterizing the system being the same as in Fig. 1. The quantity $\beta_{v v}$ is measured in units of $\left[\frac{m^{3}}{B^{2} C}\right]^{1 / 3}, \beta_{x x}$ is measured in units of $\left[\frac{C}{B}\right]^{2 / 3}$, and $\beta_{x v}$ in units of $\sqrt{m / C}[C / B]^{2 / 3}$.

for the $\beta$-parameters also describe a conservative dynamical system in the three-dimensional phase space with coordinates $\beta_{x x}, \beta_{x v}$, and $\beta_{v v}$. Indeed, if one rewrites the equations of motion (51) in a vectorial form, $d \vec{\beta} / d t=\vec{G}(\vec{\beta})$, where the $\vec{\beta}$ and $\vec{G}$ are, respectively, vectors with components $\left(\beta_{\underline{x} x}, \beta_{\underline{x}}, \beta_{v v}\right)$ and $\left(2 b \beta_{x v},-\beta_{x x}+b \beta_{v v},-2 \beta_{x v}\right)$, it is plain that $\nabla_{\beta} \cdot \vec{G}=0$, where $\vec{\nabla}_{\beta}=\left(\partial / \partial \beta_{x x}, \partial / \partial \beta_{x v}, \partial / \partial \beta_{v v}\right)$ is the $\vec{\nabla}$-operator in the $\beta$-space. In other words, the dynamics given by (51) preserves the phase-space volume in $\beta$-space. Combining the first and the third equations in (51) one finds $\dot{\beta}_{x x}+b \dot{\beta}_{v v}=0$, from which it follows that $\frac{d}{d t}\left(\beta_{x x}+b_{1} \beta_{v v}+2 b_{2} \beta_{v v}^{-1 / 2}\right)=0$, where $b_{1}=C / m$ and $b_{2}=2 \sqrt{2} \pi \frac{B \rho_{0}}{m} \Delta_{0}$. This means that, besides the integral of motion $\Delta=\beta_{x x} \beta_{v v}-\beta_{x v}^{2}$, the system (51) has a second integral of motion:

$$
\Lambda=\beta_{x x}+\frac{C}{m} \beta_{v v}+4 \sqrt{2} \pi \frac{B \rho_{0}}{m}\left(\beta_{x x} \beta_{v v}-\beta_{x v}^{2}\right) \beta_{v v}^{-1 / 2}
$$

The equations of motion (51) in $\beta$-space can be explicitly recast in terms of the integrals $\Lambda$ and $\Delta$,

$$
\frac{d \vec{\beta}}{d t}=\left(\vec{\nabla}_{\beta} \Lambda\right) \times\left(\vec{\nabla}_{\beta} \Delta\right)
$$

which means that the evolution equations for the $\beta$-parameters actually constitute a Nambu system [56,57] with invariants $\Lambda(\vec{\beta})$ and $\Delta(\vec{\beta})$.

The time evolution of the quantities $\beta_{x x}, \beta_{x v}$, and $\beta_{v v}$ in the conservative regime is illustrated in Figs. 4 and 5, where the periodic oscillatory behavior of these variables is clearly appreciable. The effective potential $\Phi(z)$ associated with the conservative regime is plotted in Fig. 6.

The conservative regime can also be analyzed using Eqs. (48) and (49), with $a=0$. The equation of motion for the variable $z=\sqrt{\beta_{v v}}$ then formally corresponds to a particle of unit mass moving in one spatial dimension under the (time-independent) potential

$$
\Phi(z)=\frac{C}{2 m} z^{2}+2 \sqrt{2} \pi \frac{B \rho_{0} \Delta}{m z} .
$$

The corresponding energy $\epsilon=\frac{1}{2} \dot{z}^{2}+\Phi(z)$ is now conserved, and $z(t)$ exhibits an oscillatory behavior around the minimum of the potential located at $z_{m}=\left(2 \sqrt{2} \pi B \rho_{0, i} \Delta_{0} / C\right)^{1 / 3}$. This, in turn, implies an oscillatory behavior for $\beta_{v v}, \beta_{x v}$, and $\beta_{x x}$. It can be verified, after some algebra, that $\epsilon=\frac{\beta_{x x}}{2}+$ $\frac{C \beta_{v v}}{2 m}+2 \sqrt{2} \pi \frac{B \rho_{0} \Delta}{m \beta_{v v}^{1 / 2}}=\frac{\Lambda}{2}$. Therefore, the conserved quantity $\epsilon$ is proportional to the integration constant $\Lambda$ that we have previously found. The conserved quantity $\epsilon$, although formally
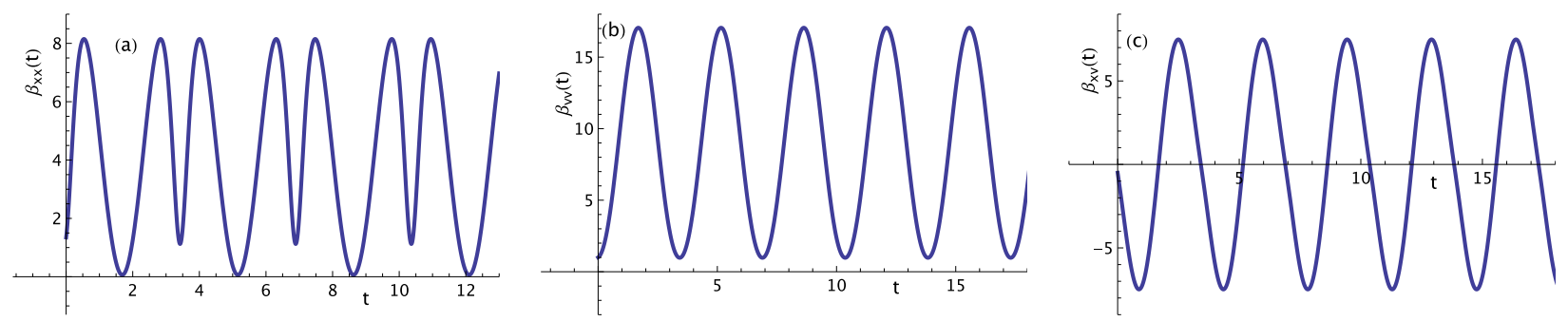

FIG. 5. Plots of $\beta_{x x}$ (a), $\beta_{v v}$ (b), and $\beta_{x v}$ (c), against time, for the conservative case. The quantity $\beta_{x x}$ is measured in units of $\left[\frac{C}{B}\right]^{2 / 3}, \beta_{v v}$ in units of $\left[\frac{m^{3}}{B^{2} C}\right]^{1 / 3}, \beta_{x v}$ in units of $\sqrt{m / C}[C / B]^{2 / 3}$, and time in units of $\sqrt{m / C}$. 


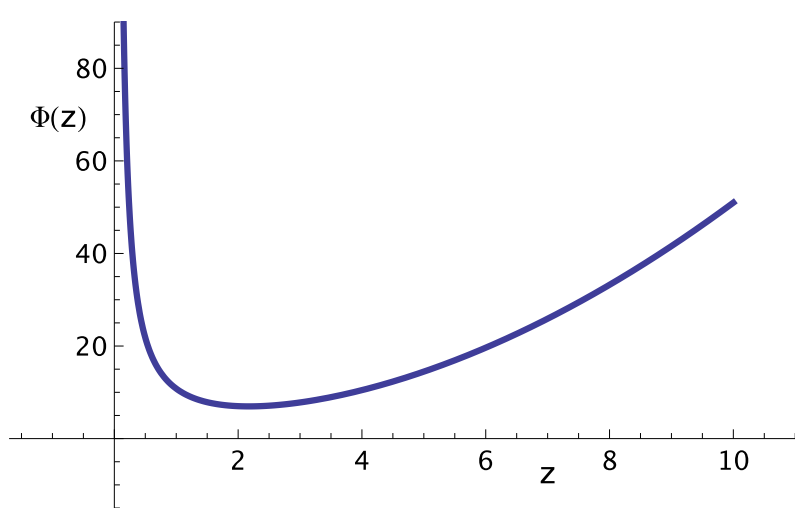

FIG. 6. Plot of the effective potential $\Phi(z)$ given by Eq. (54). The quantities $z$ and $\Phi$ are respectively measured in units of $\left[\frac{m^{3}}{B^{2} C}\right]^{1 / 6}$ and $\left[\frac{C}{B}\right]^{2 / 3}$.

corresponding to the energy associated with the $z(t)$ motion, is not equal to the physical total energy $E$ of our manyparticle system. It is, however, closely related to $E$. The physical energy is given by $E=E_{\text {kin }}+E_{\text {pot }}$, where $E_{\text {kin }}=$ $\frac{m}{2} \int v^{2} \rho(x, v, t) d x d v$ is the total kinetic energy of the manyparticle system, and $E_{\text {pot }}=\Omega+\langle W\rangle$ is the total potential energy [see Eq. (29)]. In terms of the parameters characterizing the $q$-Gaussian solution, one has

$$
\begin{aligned}
E_{\text {kin }} & =\frac{\pi \rho_{0} m v_{0}^{2}}{6 \sqrt{\Delta}}+\frac{\pi \rho_{0} m \beta_{x x}}{60 \Delta^{3 / 2}}, \\
\Omega & =\frac{\pi^{2} \sqrt{2}}{15} \frac{\rho_{0}^{2} B}{\sqrt{\beta_{v v} \Delta}}, \\
\langle W\rangle & =\frac{\pi \rho_{0} C x_{0}^{2}}{6 \sqrt{\Delta}}+\frac{\pi \rho_{0} C \beta_{v v}}{60 \Delta^{3 / 2}},
\end{aligned}
$$

and the total energy is

$$
E=N\left(\frac{m}{2} v_{0}^{2}+\frac{C}{2} x_{0}^{2}\right)+\frac{N m \Lambda}{15 \Delta},
$$

where $N=\pi \rho_{0} / 3 \sqrt{\Delta}$ is the total number of particles of the system [see (38)]. The first term in (56) constitutes the contribution to the total energy due to the position and motion of the center of mass of the system, while the second term $E_{\text {int }}=\frac{N m \Lambda}{15 \Delta}$ is the energy associated with the internal configuration and motion of the system. We see that the internal energy can be expressed in terms of the integration constants $\Lambda$ and $\Delta$.

The equations of motion (50) and (51) admit a stationary solution corresponding to $x_{0}=0, v_{0}=0, \beta_{x v}=0$, and $\beta_{x x}=$ $b \beta_{v v}$. The corresponding stationary solution of the Vlasov equation is then

$$
\rho_{\mathrm{st}}(x, v)=\rho_{0}\left\{1-4 \beta_{v v}\left[\frac{v^{2}}{2}+\phi_{\mathrm{sp}}(x)\right]\right\}_{+},
$$

where

$$
\phi_{\mathrm{sp}}(x)=\left[\frac{1}{2} \frac{C}{m}-\sqrt{2} \pi \frac{B \rho_{0}}{m \sqrt{\beta_{v v}}} \beta_{x x}\right] x^{2}
$$

is the potential field (per unit mass) experienced by one single particle of the system (the subindex "sp" stands for "single particle"). The first term within the square bracket in the right-hand side of (58) corresponds to the contribution to $\phi_{\mathrm{sp}}(x)$ due to the external confining potential, while the second term arises from the interaction of the test particle with the rest of the particles of the system. We see that the stationary space-velocity distribution depends on the $(x, v)$ variables only through the single-particle energy per unit mass, $\varepsilon=\frac{v^{2}}{2}+\phi_{\mathrm{sp}}(x)$, which is an integral of motion of the individual particles. This is in agreement with Jean's theorem [35], which states that stationary solutions of the Vlasov equation depend on the space-velocity variables only through integrals of motion of the individual particles. It is worth mentioning that the stationary space-velocity density actually has a $q$-exponential dependence on the single-particle energy, $\rho_{\mathrm{st}}=\rho_{0}\left[1-\left(1-q^{\prime}\right) 2 \beta_{v v} \varepsilon\right]_{+}^{1 /\left(1-q^{\prime}\right)}$, with $q^{\prime}=-1$.

\section{CONCLUSIONS}

We have investigated the main properties of a Vlasov dynamics arising from the incorporation of inertial effects to a many-body system described by a nonlinear Fokker-Planck equation. The nonlinear Fokker-Planck equation with a powerlaw nonlinearity in the diffusion term describes a system of particles interacting via short-range forces and moving, in an overdamped regime, under the effects of an external confining potential. By including inertial effects, the nonlinear Fokker-Planck dynamics governing the evolution of the space distribution of particles becomes embedded within a Vlasov dynamics governing the space-velocity distribution of particles. This Vlasov dynamics leads to a reinterpretation of the $H$ theorem satisfied by the nonlinear Fokker-Planck equation. The free-energy-like quantity associated with these $H$ theorems coincides with the total potential energy of the many-body system under consideration, which comprises two terms: one associated with the interaction between the particles, and the other one associated with the external confining potential. The first term corresponds to the (generalized) entropic functional appearing in the aforementioned free-energy-like quantity. We have obtained a family of exact, analytical time-dependent solutions for the Vlasov equation exhibiting the form of time-dependent $q$-Gaussian space-velocity densities. These solutions involve two $q$-values related by a simple equation. One $q$-value characterizes the space-velocity distribution and the other one the (velocity-integrated) spatial density. The occurrence of more than one, interrelated, $q$-values is a pattern also observed in other applications of the $S_{q}$ thermostatistics [54,55].

Research conducted in recent years indicates that the $S_{q}$ thermostatistics helps to understand the thermodynamics of confined, interacting particles under the effect of drag forces in the overdamped limit $[16,32,33]$. Important applications, such as the study of vortices in type II superconductors [7,10], attest to that. The main aim of the present effort was to relax the overdamped motion assumption in order to incorporate inertial effects within the aforementioned scenarios. These developments enlarge the range of possible applications of the $S_{q}$ thermostatistical approach to other systems of confined particles affected by drag forces. This is specially relevant 
for systems constituted by particles that, unlike the type II superconducer vortices, have finite (nonvanishing) mass. Interesting candidates for future applications are systems of particles interacting via Yukawa potentials, used for modeling dusty and complex plasmas [58-62].

\section{ACKNOWLEDGMENTS}

We acknowledge partial financial support from the Brazilian science funding agencies $\mathrm{CNPq}$ and FAPERJ. C.T. also acknowledges partial financial support from the John Templeton Foundation (USA).
[1] M. S. Ribeiro, G. A. Casas, and F. D. Nobre, Phys. Rev. E 91, 012140 (2015).

[2] Z. G. Arenas, D. Barci, and T. Tsallis, Phys. Rev. E 90, 032118 (2014).

[3] L. C. Malacarne, R. S. Mendes, I. T. Pedron, and E. K. Lenzi, Phys. Rev. E 65, 052101 (2002).

[4] E. K. Lenzi, C. Anteneodo, and L. Borland, Phys. Rev. E 63, 051109 (2001).

[5] M. Shiino, J. Math. Phys. 42, 2540 (2001).

[6] T. D. Frank, Physica A 310, 397 (2002).

[7] J. S. Andrade, Jr., G. F. T. da Silva, A. A. Moreira, F. D. Nobre, and E. M. F. Curado, Phys. Rev. Lett. 105, 260601 (2010).

[8] R. S. Wedemann, A. R. Plastino, and C. Tsallis, Phys. Rev. E 94, 062105 (2016).

[9] T. D. Frank, Nonlinear Fokker-Planck Equations: Fundamentals and Applications (Springer, Berlin, 2005).

[10] M. S. Ribeiro, F. D. Nobre, and E. M. F. Curado, Phys. Rev. E 85, 021146 (2012).

[11] G. Combe, V. Richefeu, M. Stasiak, and A. P. F. Atman, Phys. Rev. Lett. 115, 238301 (2015).

[12] M. Shiino, Phys. Rev. E 67, 056118 (2003).

[13] P. H. Chavanis, Phys. Rev. E 68, 036108 (2003).

[14] T. D. Frank and R. Friedrich, Physica A 347, 65 (2005).

[15] M. R. Ubriaco, Phys. Lett. A 373, 4017 (2009).

[16] E. M. F. Curado, A. M. C. Souza, F. D. Nobre, and R. F. S. Andrade, Phys. Rev. E 89, 022117 (2014).

[17] J. C. Fores, Physica A 467, 432 (2017).

[18] G. A. Mendes, M. S. Ribeiro, R. S. Mendes, E. K. Lenzi, and F. D. Nobre, Phys. Rev. E 91, 052106 (2015).

[19] P. H. Chavanis, Eur. Phys. J. B 62, 179 (2008).

[20] M. Mulansky and A. Pikovsky, New J. Phys. 15, 053015 (2013).

[21] V. Schwämmle, E. M. F. Curado, and F. D. Nobre, Eur. Phys. J. B 58, 159 (2007).

[22] V. Schwämmle, F. D. Nobre, and E. M. F. Curado, Phys. Rev. E 76, 041123 (2007)

[23] C. Tsallis, J. Stat. Phys. 52, 479 (1988).

[24] M. A. F. dos Santos and E. K. Lenzi, Phys. Rev. E 96, 052109 (2017).

[25] C. Tsallis, Introduction to Nonextensive Statistical Mechanics (Springer, New York, 2009).

[26] C. Beck, Contemp. Phys. 50, 495 (2009).

[27] F. A. Bais and J. D. Farmer, in Handbook of Philosophy of Information, edited by P. W. Adriaans and J. F. A. K. van Benthem (Elsevier Science Publishers, Amsterdam, The Netherlands, 2008), pp. 609-684.

[28] A. Pluchino and A. Rapisarda, Physica A 365, 184 (2006).

[29] A. R. Plastino and A. Plastino, Physica A 222, 347 (1995).

[30] J. M. Conroy and H. G. Miller, Phys. Rev. E 91, 052112 (2015).

[31] S. Asgarani, Phys. Rev. E 91, 022104 (2015).

[32] M. S. Ribeiro and F. D. Nobre, Phys. Rev. E 94, 022120 (2016).
[33] C. M. Vieira, H. A. Carmona, J. S. Andrade, Jr., and A. A. Moreira, Phys. Rev. E 93, 060103(R) (2016).

[34] C. Tsallis and D. J. Bukman, Phys. Rev. E 54, R2197(R) (1996).

[35] J. Binney and S. Tremaine, Galactic Dynamics (Princeton University Press, Princeton, NJ, 2008).

[36] Y. L. Klimontovich, The Statistical Theory of Non-Equilibrium Processes in a Plasma (Pergamon Press, Oxford, 1967).

[37] J. H. Jeans, Problems of Cosmology and Stelar Dynamics (Cambridge University Press, Cambridge, 1919).

[38] A. A. Vlasov, Zh. Eksp. Teor. Fiz. 8, 291 (1938).

[39] J. A. S. Lima, R. Silva, and J. Santos, Astron. Astrophys. 396, 309 (2002).

[40] V. F. Cardone, M. P. Leubner, and A. Del Popolo, MNRAS 414, 1365 (2011).

[41] J. Calvo, E. Florido, O. Sanchez, E. Battaner, J. Soler, and B. Ruiz-Granados, Physica A 388, 2321 (2009).

[42] T. Kronberger, M. P. Leubner, and E. van Kampen, Astron. Astrophys. 453, 21 (2006).

[43] C. Bardos and A. Nouri, J. Math. Phys. 53, 115621 (2012).

[44] V. Baran, M. Colonna, M. Di Toro, B. Frecus, A. Croitoru, and D. Dumitru, Eur. Phys. J. D 68, 356 (2014).

[45] A. Patelli and S. Ruffo, Eur. Phys. J. D 68, 329 (2014).

[46] C. Vignat, A. Plastino, and A. R. Plastino, Physica A 390, 2491 (2011).

[47] L. J. L. Cirto, V. R. V. Assis, and C. Tsallis, Physica A 393, 286 (2014).

[48] H. Christodoulidi, T. Bountis, C. Tsallis, and L. Drossos, J. Stat. Mech. (2016) 123206.

[49] L. Ferrari, Chem. Phys. 336, 27 (2007).

[50] L. Ferrari, Chem. Phys. 428, 144 (2014).

[51] M. Hohmann, F. Kindermann, T. Lausch, D. Mayer, F. Schmidt, E. Lutz, and A. Widera, Phys. Rev. Lett. 118, 263401 (2017).

[52] C. Tsallis, M. Gell-Mann, and Y. Sato, Proc. Natl. Acad. Sci. USA 102, 15377 (2005).

[53] C. Tsallis, Eur. Phys. J. Special Topics 226, 455 (2017).

[54] C. Tsallis, in Physical and Mathematical Aspects of Symmetries, Proceedings of the 31st International Colloquium in Group Theoretical Methods in Physics, edited by S. Duarte, J.-P. Gazeau, S. Faci, T. Micklitz, R. Scherer, and F. Toppan (Springer, Cham, Switzerland, 2017), pp. 51-60.

[55] A. A. Tateishi, R. Hanel, and S. Thurner, Phys. Lett. A 377, 1804 (2013).

[56] Y. Nambu, Phys. Rev. D 7, 2405 (1973).

[57] M. Axenides and E. Floratos, J. High Energy Phys. 04 (2010) 036.

[58] R. T. Farouki and S. Hamaguchi, J. Chem. Phys. 101, 9885 (1994).

[59] P. K. Shukla, Phys. Plasmas 8, 1791 (2001).

[60] B. Liu and J. Goree, Phys. Rev. Lett. 100, 055003 (2008).

[61] T. E. Sheridan, Phys. Plasmas 16, 083705 (2009).

[62] S. A. Khrapak, O. S. Vaulina, and G. E. Morfill, Phys. Plasmas 19, 034503 (2012). 